\title{
Padrões de consumo de energia no sector residencial: uma revisão bibliográfica
}

\section{Manuel Tomás Manuel ${ }^{1}$ e Alfiado Victorino ${ }^{2}$}

${ }^{1}$ Universidade Licungo. Faculdade de Ciências e Tecnologia. Curso de Física. Extensão da Beira. Moçambique.E-mail: mtmanuel136@gmail.com.

${ }^{2}$ Universidade Licungo. Faculdade de Ciências e Tecnologia. Extensão da Beira. Moçambique.

Resumo. O presente artigo tem como objectivo analisar as produções científicas nacionais e internacionais, que abordam a temática dos padrões de consumo de energia no sector residencial como referência para estudo dos padrões de consumo doméstico de energia no Bairro Nhaconjo, Cidade da Beira, no centro de Moçambique. Para tal analisamos cerca de 26 artigos científicos publicados em revistas científicas indexadas, nas últimas três décadas. Os resultados demonstram a existência de muitos estudos que tratam dos padrões de consumo doméstico de energia em países desenvolvidos, porém pouca literatura trata do assunto referente a Moçambique. A literatura revista também se mostra enfática em relação as diferenças em termos de agregados familiares, nível de rendimento, idade, escolaridade, hábitos do usuário, entre outros factores que influenciam directamente os comportamentos relacionados com o consumo de energia.

Palavras-chave: Padrões de consumo; Energia; Sector doméstico; Moçambique.

\begin{abstract}
Energy consumption standards in the residential sector: A bibliographic review. This paper aims to analyze the national and international scientific productions, which address the theme of energy consumption patterns in the residential sector as a reference for the study of domestic energy consumption patterns in Nhaconjo Neighborhood, Beira City, central Mozambique. For this we analyzed about 26 scientific articles published in indexed scientific journals in the last three decades. The results show that there are many studies that address domestic energy consumption patterns in developed countries, but little literature on the subject of Mozambique. The revised literature is also emphatic regarding differences in household levels, income level, age, education, user habits, among other factors that directly influence behaviors related to energy consumption.
\end{abstract}

Keywords: Consumption standards; Energy; Domestic sector; Mozambique.

Recebido

$22 / 03 / 2020$

Aceito

$26 / 07 / 2020$

Disponível on line

$30 / 07 / 2020$

Publicado

$31 / 08 / 2020$

Acesso aberto

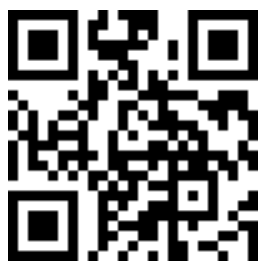

ORCID

(D) 0000-0001-5101-6326 Manuel Tomás Manuel

(ㄷ) 0000-0003-3134-3607 Alfiado Victorino

ISSN 2359-1412/RBGAS-2020-0057/2020/7/16/10/605

Rev. Bras. Gest. Amb. Sustent.

http://revista.ecogestaobrasil.net 


\begin{abstract}
Resumen. Normas de consumo de energía en el sector residencial: Una revisión bibliográfica. Este documento tiene como objetivo analizar las producciones científicas nacionales e internacionales, que abordan el tema de los patrones de consumo de energía en el sector residencial como referencia para el estudio de los patrones de consumo de energía doméstica en el barrio de Nhaconjo, ciudad de Beira, en el centro de Mozambique. Para esto analizamos alrededor de 26 artículos científicos publicados en revistas científicas indexadas en las últimas tres décadas. Los resultados muestran que hay muchos estudios que abordan los patrones de consumo de energía doméstica en los países desarrollados, pero poca literatura sobre el tema de Mozambique. La literatura revisada también es enfática con respecto a las diferencias en los niveles de los hogares, el nivel de ingresos, la edad, la educación, los hábitos de los usuarios, entre otros factores que influyen directamente en los comportamientos relacionados con el consumo de energía.
\end{abstract}

Palabras clave: Normas de consumo; Energía; Sector doméstico; Mozambique.

\title{
Introdução
}

O consumo é um fenómeno social e denso que atravessa diferentes dimensões da experiência humana (Rocha e Barros, 2008; Abrão, 2008) envolvendo a utilização, aplicação, ou uso de um certo bem por um indivíduo, comunidade ou uma nação (Dominik, 2010). Padrões de consumo doméstico de energia estão intimamente relacionados com a forma como as famílias usam a energia para suprir suas necessidades energéticas.

Segundo Druckman e Jackson (2009), o consumo residencial de energia representa suprimentos energéticos usados em edificações de carácter residencial assim como no terreno onde a mesma esteja localizada. Subentende-se que a energia seja consumida directamente para fins residenciais, isto é, para funcionamento dos electrodomésticos e de todos os equipamentos que venham a fazer parte do funcionamento da edificação em si, excluindo-se aquela consumida indirectamente.

A energia é um factor crucial para o desenvolvimento de um país e é usada para vários fins a nível industrial, comercial e doméstico. 0 consumo de energia é um dos principais indicadores do desenvolvimento económico e do nível de qualidade de vida de qualquer sociedade (Sebastião, 2013; Silva, 2016; Burgsdorff, 2017). 0 uso deste insumo não só tem reflexos no ritmo de actividade dos sectores supracitados, bem como nos recursos que as famílias podem dedicar ao consumo de energia. 0 sector doméstico é um dos segmentos importantes no uso de energia, pois, é um dos que mais consome energia disponível em comparação com outros segmentos sociais e, estudos feitos indicam que o consumo deste sector tende a subir (Dergiades e Tsoulfidis, 2011; Cebula, 2012; Bekhet e Ivy-Yap, 2014; Mahumane e Mulder, 2015). Esta subida está associada a vários factores de entre os quais podemos citar por exemplo, o crescimento demográfico bem como o aumento do poder de compra que por sua vez contribui para elevação dos padrões de conforto térmico, iluminação, lazer, aquisição de equipamentos, entre outros benefícios.

Moçambique enfrenta ainda o problema de acesso (limitado) a produtos e serviços energéticos modernos, dado que, maior parte da sua população ainda depende da biomassa tradicional (Burgsdorff, 2017). Com efeito, dados disponíveis indicam que mais de $79,2 \%$ da população depende da biomassa para satisfazer as necessidades energéticas 
(Sebastião, 2013; Burgsdorff, 2017; Manuel e Victorino, 2020a, b). No entanto, o crescimento da população, bem como os esforços de desenvolvimento em curso no país vêm ditando o aumento do consumo de energia, em particular aquela proveniente da biomassa e dos combustíveis fósseis (petróleo, gás natural e carvão mineral).

Este aumento do consumo de energia poderá impactar seriamente na segurança energética, qualidade ambiental, mudanças climáticas, assim como na saúde pública (Ding et al., 2017).

Por conseguinte, o estudo dos padrões de consumo de energia na perspectiva do estilo de vida dos consumidores do sector doméstico se apresenta como parte de uma estratégia de desenvolvimento nacional fundada nos princípios da sustentabilidade e no uso racional e equilibrado dos recursos energéticos disponíveis no país.

Neste sentido, o presente artigo de revisão bibliográfica tem como objectivo principal o levantamento e análise (estado de arte) dos estudos feitos sobre padrões de consumo doméstico de energia em vários contextos, como uma primeira e modesta contribuição no entendimento das dinâmicas envolvidas no consumo de energia nas comunidades moçambicanas, com foco na Cidade da Beira, particularmente no $14^{\circ}$ Bairro Nhaconjo.

\section{Materiais e métodos}

O presente trabalho consiste na revisão bibliográfica sobre padrões de consumo doméstico de energia. Para a sua concretização fez-se uma busca em diferentes fontes que incluem livros, artigos científicos, teses e sites específicos, usando os seguintes descritores, "Padrões de consumo" "Energia" e "Sector doméstico". As referências bibliográficas dos estudos localizados foram também rastreadas para localizar outros trabalhos pertinentes ao assunto abordado nesse artigo. 0 levantamento bibliográfico totalizou 54 publicações, das quais 35 pesquisas foram seleccionadas, entre estes 26 são artigos científicos. Foi realizada uma análise de títulos e resumos para obtenção de artigos, teses, entre outros documentos potencialmente relevantes para a revisão. Pesquisas relacionados à intervenção realizada totalmente fora dos padrões de consumo doméstico de energia (agricultura, transportes, indústrias) foram excluídos da análise. Para delimitação temporal a revisão da literatura em causa limitou-se aos trabalhos publicados desde a década de 1990 até os dias de hoje.

\section{Resultados e discussão}

\section{Caracterização do acervo revisado}

Os resultados apresentados nesta secção são referentes a uma série de estudos analisados que tratam dos padrões de consumo de energia no sector doméstico. Durante a revisão da literatura alguns aspectos foram identificados como relevantes no processo de análise dos conteúdos. Tendo em conta o objectivo de cada publicação, os trabalhos analisados foram agrupados em dois eixos temáticos, a saber:

(1) Abordagem conceitual que inclui conceito de padrão de consumo de energia, combustíveis e seu uso final no sector doméstico, factores e impactos associados aos usos finais dos combustíveis energéticos no sector residencial; e

(2) Estado da arte sobre padrões de consumo doméstico de energia.

A Tabela 1 ilustra a caracterização do acervo estudado, segundo o autor, ano da publicação, natureza da pesquisa, local do estudo, eixo temático e instrumento metodológico utilizado. 
Tabela 1. Pesquisas relacionadas.

\begin{tabular}{|c|c|c|c|c|c|}
\hline Autores & Ano & Natureza & $\begin{array}{c}\begin{array}{c}\text { Local do } \\
\text { estudo }\end{array} \\
\end{array}$ & $\begin{array}{c}\text { Eixo } \\
\text { temático }\end{array}$ & Instrumentos \\
\hline Eberhard & 1990 & Artigo & África do Sul & 2 & Questionário \\
\hline Cohen & 2002 & Tese & Brasil & 1 & Análise da situação \\
\hline Tso e Yau & 2003 & Artigo & Hong Kong & 2 & Questionário \\
\hline Achão & 2003 & Tese & Brasil & 2 & Revisão bibliográfica \\
\hline Bethonico & 2006 & Artigo & Brasil & 1 & Questionário \\
\hline ELETROBRAS & 2007 & Manual & Brasil & 1 & Questionários/entrevista \\
\hline Rocha e Barros & 2008 & Artigo & Brasil & 1 & Orçamento familiar (POF) \\
\hline Comini et al. & 2008 & Manual & Portugal & 1 & ------- \\
\hline Abrão & 2008 & Artigo & Brasil & 1 & Revisão bibliográfica \\
\hline $\begin{array}{l}\text { Druckman e } \\
\text { Jackson }\end{array}$ & 2009 & Artigo & Reino Unido & -- & Questionário \\
\hline Cortez & 2009 & Artigo & Brasil & 1 & Revisão bibliográfica \\
\hline Tódero & 2009 & Tese & Brasil & 1 & Questionário \\
\hline Carvalho e Alves & 2010 & Artigo & Brasil & 1 & Orçamento familiar \\
\hline Mendes & 2010 & Artigo & Portugal & 1 & Revisão Bibliográfica \\
\hline $\begin{array}{l}\text { Dergiades e } \\
\text { Tsoulfidis }\end{array}$ & 2011 & Artigo & Grécia & -- & Censo demográfico \\
\hline Arthur et al. & 2011 & Relatório & Moçambique & 1 & Revisão bibliográfica \\
\hline Kowsari e Zerriffi & 2011 & Artigo & África do Sul & 1 & Questionário \\
\hline $\begin{array}{l}\text { Ministério da } \\
\text { Energia }\end{array}$ & 2011 & $\begin{array}{c}\text { Manual } \\
\text { (ENDER) }\end{array}$ & Moçambique & 1 & Análise da situação \\
\hline Yohanis & 2012 & Artigo & Reino Unido & 2 & Questionário \\
\hline Sousa et al. & 2012 & Artigo & Portugal & 1 & Revisão bibliográfica \\
\hline Cebula & 2012 & Artigo & EUA & -- & Dados estatísticos \\
\hline Arthur et al. & 2012 & Artigo & Moçambique & 1 & Revisão bibliográfica \\
\hline Dominik et al. & 2012 & Artigo & Brasil & 1 & Questionário \\
\hline Canettieri et al. & 2013 & Artigo & Brasil & 1 & Entrevista \\
\hline Oliveira et al. & 2013 & Artigo & Brasil & -- & Revisão bibliográfica \\
\hline Bekhet e Ivy-Yap & 2014 & Artigo & Malásia & -- & Questionário \\
\hline La Rovere & 2015 & Artigo & $\begin{array}{l}\text { Dinamarca, } \\
\text { Reino Unido }\end{array}$ & 1 & Revisão bibliográfica \\
\hline Ofetotse et al. & 2015 & Artigo & Botswana & 2 & Questionário \\
\hline Ferreira & 2015 & Tese & Brasil & 1 & $\begin{array}{c}\text { Software Matlab (toolbox), } \\
\text { Java }\end{array}$ \\
\hline $\begin{array}{l}\text { Mahumane e } \\
\text { Mulder }\end{array}$ & 2015 & Artigo & Moçambique & -- & $\begin{array}{c}\text { Revisão bibliográfica e } \\
\text { Entrevista }\end{array}$ \\
\hline Burgsdorff & 2017 & Relatório & Moçambique & 1 & Consulta bibliográfica \\
\hline Silva et al. & 2017 & Artigo & Brasil & -- & Questionário \\
\hline Ateba et al. & 2018 & Artigo & África do Sul & 2 & Questionários \\
\hline Manuel e Victorino & $2020 \mathrm{a}$ & Artigo & Moçambique & -- & Questionário \\
\hline Manuel e Victorino & $2020 \mathrm{~b}$ & Artigo & Moçambique & -- & Questionário \\
\hline
\end{tabular}

Fonte: Adaptado de Oliveira et al. (2013).

Uma análise preliminar da bibliografia revista mostra uma grande diversidade em termos da natureza da literatura revista (artigos, teses, manuais, entre outros materiais). É igualmente notória a diversidade em termos de origem dos trabalhos, no entanto, registase uma certa predominância de trabalhos de origem brasileira. 0 ano predominante foi 2012, com cinco artigos, seguida dos anos 2009, 2011 e 2015 com quatro pesquisas para cada ano, totalizando oito artigos, duas teses, um relatório e um manual. 0 idioma 
predominante foi a língua portuguesa com 21 pesquisas publicadas. Os demais (5) foram publicados na língua inglesa.

Em relação aos instrumentos usados destaca-se o questionário, dirigido aos residentes, com base em revisões bibliográficas e em pesquisas de campo ou estudos de caso. Entretanto, segundo Moura et al. (1998) "estes métodos são destacados por possuir a capacidade de reunir informações sobre percepções, crenças e opiniões dos indivíduos estudados", muito determinantes nos padrões de consumo doméstico de energia.

Um dado importante a reter é o reduzido número de trabalhos publicados internamente, que versam sobre os padrões de consumo de energia doméstica em Moçambique. A falta de estudos desta natureza no país, poderá dificultar acções orientadas para o combate da pobreza energética, desenvolvimento de acções de eficiência energética, entre outras actividades necessárias para assegurar o uso sustentável dos recursos energéticos disponíveis no país. Por conseguinte, o presente artigo pretende cobrir parcialmente essa lacuna analisando os padrões de consumo doméstico de energia da população moçambicana, tomando como referência os moradores do $14^{\circ}$ Bairro Nhaconjo, da Cidade da Beira, Zona Central de Moçambique.

\section{Conceito de padrão de consumo de energia}

Segundo o dicionário Priberam padrão ou paradigma é o que serve de referência ou de modelo, o termo equivalente em inglês (pattern) significa uma maneira particular pela qual algo é feito, organizado ou acontece. Portanto, no contexto deste artigo, padrões de consumo doméstico de energia, se referem à maneira particular como os cidadãos de uma certa região utilizam determinados combustíveis/fontes de energia para satisfação das suas necessidades energéticas. Tornando-os deste modo, importantes fontes estratégicas de informação sobre o consumo de tais combustíveis energéticos numa determinada comunidade.

Nesta perspectiva, os padrões de consumo podem ser abordados em quatro dimensões a saber, económico (Silva, 2016; Werlang e Geller, 2018), político (Cohen, 2002), sociológico (Abrão, 2008; Dominik, 2010) e ambiental (IAEA, 2005).

No âmbito económico o padrão de consumo energético é um indicador importante do modo de desenvolvimento e do crescimento económico de uma sociedade (Sebastião, 2013; Silva, 2016; Werlang e Geller, 2018). Com efeito, o crescimento económico dos países depende fortemente da oferta de suprimentos adequados e confiáveis de energia, seja para aumentar a produtividade, ou para garantir melhor qualidade de vida.

Do ponto de vista sociológico o padrão de consumo energético é definido como um valor simbólico importante (Abrão, 2008; Dominik, 2010), pois, o consumo torna-se um factor de distinção, ele hierarquiza e classifica, conferindo status em função da qualidade e quantidade de energia usada entre membros de uma mesma comunidade ou sociedade. 0 acesso à energia tem sido utilizado como indicador de nível de pobreza da população e como factor de inclusão e diferenciação social, visto que, o uso energético é diferente entre ricos e pobres (Kemmler e Spreng, 2007; Goldemberg e Lucon, 2007) e, "através dos objectos, ou melhor, do consumo, o indivíduo se encontrará em um determinado grupo e, ao mesmo tempo, se distanciará da imagem dos grupos que não pertence" (Abrão, 2008, p. 50).

E, no âmbito político o padrão de consumo de energia é visto como uma das medidas de garantir a eficácia das políticas destinadas a conservação de energia nos agregados familiares; encontrapartida, é visto como medida do nível de acesso aos bens e serviços (Cohen, 2002), isto é, reflecte a estrutura espacial, educacional e política da sociedade. Por fim, na dimensão ambiental, o padrão de consumo de energia é visto como uma das fontes de pressão sobre o meio ambiente local e global (IAEA, 2005). Visto que, a 
dimensão dos impactos ambientais depende, em grande medida, de quanta energia é produzida e consumida bem como do tipo de energia usada.

\section{Combustíveis energéticos usados no sector residencial}

Em relação as fontes de energia utilizadas, Scari (2011) analisando o consumo de energia no sector residencial brasileiro, divide-as em fontes directas (gás natural, carvão fóssil, lenha, bagaço de cana, etc.) e secundárias (electricidade, coque de carvão fóssil, carvão vegetal, álcool etílico, gás de coque e outras). No mesmo diapasão, Ferreira (2015) e Roger e Merlin (2010) classificam as fontes de energia em duas categorias, renováveis e não renováveis.

Num estudo sobre estimativa das elasticidades para a demanda de energia doméstica em Moçambique, Arthur et al. (2012) consideram que, as famílias moçambicanas consomem uma mistura de combustíveis energéticos para satisfazer as suas necessidades. Para estes autores, o uso diversificado de energia doméstica depende dos preços dos combustíveis e da capacidade da família para investir nos electrodomésticos. Com base em dados de uma pesquisa domiciliar realizada em Moçambique pelo Instituto Nacional de Estatística (INE) entre 2002 e 2003, estes autores calculam o preço e as elasticidades da renda e da demanda de energia doméstica, usando um método econométrico desenvolvido por Deaton3. Os resultados obtidos neste estudo, sugerem que no contexto moçambicano, os combustíveis energéticos dominantes são, electricidade, lenha, GLP, gás natural, carvão vegetal e querosene.

0 mesmo resultado pode ser encontrado em vários trabalhos sobre o uso doméstico de energia em Moçambique (Arthur et al., 2011; Sousa et al., 2012; Burgsdorff, 2017). Os resultados destes autores sugerem que as fontes de energia mais usadas nas famílias moçambicanas são biomassa (lenha, carvão vegetal), querosene, gás natural (GLP), electricidade, petróleo, fotovoltaica, baterias e gerador. Note-se, no entanto, que a classificação elaborada pelos autores supracitados não oferece uma imagem completa e fidedigna da matriz energética moçambicana, pois, exclui outras fontes usadas com frequência pelas comunidades deste país, particularmente no meio rural e peri-urbano, pertencentes a categoria de biomassa tradicional, tais como, excremento bovino, diferentes tipos de palhas, serradura, etc.

\section{Usos finais de combustíveis energéticos no sector residencial}

Relativamente ao uso final para qual os combustíveis energéticos têm sido aplicados, a literatura revista mostra que, em Portugal, por exemplo os consumos de energia nos edifícios residenciais distribuem-se aproximadamente da seguinte forma: cozinhas e águas quentes sanitárias 50\%, aquecimento e arrefecimento $25 \%$, iluminação e equipamentos electrodomésticos $25 \%$ (Comini et al., 2008). Sem quantificar, o manual da ELETROBRAS (2007), apresenta os sectores do uso final de energia no sector doméstico brasileiro, constituído por aquecimento de água, condicionamento ambiental, conservação de alimentos, iluminação, lazer, serviços gerais, como ferro eléctrico, máquina de lavar roupa, microondas e outros (liquidificador, batedeira, aspirador de pó, etc.).

No contexto moçambicano, os usos finais identificados por Arthur et al. (2011), como de maior inserção no sector residencial são: iluminação, comunicação, refrigeração, condicionamento ambiental, aquecimento e cozimento ou cocção. Ainda nesse contexto, de acordo com o Ministério da Energia (2011), as necessidades básicas a satisfazer pelos fornecimentos de energia nas populações moçambicanas incluem aquelas dos agregados familiares, das infra-estruturas comunitárias, de instituições de serviço público e de estabelecimentos comerciais e industriais, conforme ilustra a Tabela 2. 
Tabela 2. Aplicações energéticas típicas de Moçambique.

\begin{tabular}{|c|c|c|c|}
\hline Aplicações energéticas & Famílias & $\begin{array}{l}\text { Comunidade e } \\
\text { Serviço Público }\end{array}$ & $\begin{array}{l}\text { Comércio e } \\
\text { Indústria }\end{array}$ \\
\hline Iluminação & $\mathrm{x}$ & $\mathrm{x}$ & $\mathrm{x}$ \\
\hline Confecção de alimentos & $\mathrm{x}$ & $\mathrm{x}$ & \\
\hline Refrigeração & $\mathrm{x}$ & $\mathrm{x}$ & $\mathrm{x}$ \\
\hline Aparelhos/Entretenimento & $\mathrm{x}$ & $\mathrm{x}$ & $\mathrm{x}$ \\
\hline Iluminação pública & $\mathrm{x}$ & $\mathrm{x}$ & \\
\hline Aquecimento de água & $\mathrm{x}$ & $\mathrm{x}$ & $\mathrm{x}$ \\
\hline Uso produtivo (geração de rendimentos) & $\mathrm{x}$ & $\mathrm{x}$ & $\mathrm{x}$ \\
\hline Comunicação & $\mathrm{x}$ & $\mathrm{x}$ & $\mathrm{x}$ \\
\hline Meios de transporte & $\mathrm{x}$ & $\mathrm{x}$ & $\mathrm{x}$ \\
\hline Bombeamento de água & & $\mathrm{x}$ & \\
\hline Outros & $\mathrm{x}$ & $\mathrm{x}$ & $\mathrm{x}$ \\
\hline
\end{tabular}

Fonte: Ministério da Energia (2011).

De acordo com a tabela acima percebe-se que a maior parte das aplicações energéticas referidas dizem respeito ou estão ligadas ao consumo doméstico, o que mostra a importância, pertinência e relevância deste sector na matriz energética moçambicana.

\section{Factores que condicionam os padrões de consumo doméstico de energia}

No intuito de identificar factores que condicionam os padrões de consumo de energia nos diferentes sectores, autores que se debruçam sobre o consumo de energia no sector residencial mostram que a distribuição do consumo de energia está relacionada a vários factores, tais como estilo de vida e estruturas familiares5 que ocorrem na sociedade (Carvalho e Alves, 2010). Além dos factores mencionados, Dominick et al. (2012), Schaffrin e Reibling (2015) apontam outros como, económicos, psicológicos, sociológicos e nível de renda que afectam o comportamento do consumidor. A demanda para cada fonte de energia depende também de seu potencial para a prestação de serviços e das preferências do agregado familiar em particular. Para Vasconcelos (2002) os factores determinantes incluem o preço dos combustíveis e dos bens, clima, propaganda, hábito dos consumidores, entre outros.

Em outras circunstâncias, Castro (2008), Blackwell et al. (2009), Veiga Neto et al. (2015) destacam a importância dos aspectos culturais na formação dos padrões de consumo. Sem mencionar exactamente quais e nem o contexto, Kowsari e Zerriffi (2011) falam em factores endógenos e exógenos na escolha de combustível nas famílias.

\section{Impactos associados aos usos finais dos combustíveis no sector residencial}

Quanto aos impactos associados ao uso final dos combustíveis energéticos no sector residencial, Cortez (2009) destaca que, pela sua natureza, a sociedade de consumo exige cada vez mais conforto, o que implica maior geração, transmissão, distribuição e uso de energia, gerando impactos negativos à saúde humana e ao meio ambiente, tais como modificação da paisagem e do clima, alterações dos ecossistemas, da fauna, flora, entre outros. Bethonico (2006), Nkundumukiza (2009) e Canettieri et al. (2013) analisam os impactos socioambientais causados pelo uso do carvão vegetal nas famílias brasileiras, tendo constatado que a produção e o uso deste combustível promove profundas alterações ambientais e sociais, gerando sérios problemas respiratórios como a asma, diminuição da função respiratória, além de irritação ocular que pode levar a catarata. A título de exemplo, os estudos feitos mostram que o desmatamento acentuado e emissões de $\mathrm{CO}_{2}$ produziram modificações não apenas na paisagem, mas na vida quotidiana de várias regiões do Brasil.

Já o uso de combustíveis não renováveis (o carvão mineral, o petróleo, o gás natural, etc.), representam elevados impactos ambientais vinculados à poluição 
atmosférica e o consequente aquecimento global, podendo contribuir para a destruição da camada de ozono, para o efeito estufa, chuva ácida, mudanças climáticas entre outros efeitos (Barquete e Silva, 2013).

Portanto, do levantamento feito observa-se que os factores determinantes na formação dos padrões de consumo das famílias são dinâmicos, diversos e multidimensionais, dai a necessidade de estudos de campo/caso para o entendimento das dinâmicas envolvidas na construção desses padrões.

\section{Estado da arte sobre padrões de consumo de energia no sector residencial}

Em termos de estado da arte relativa aos padrões de consumo doméstico de energia, a revisão de literatura feita neste artigo mostra que desde a década 1990, uma maior atenção é dada a optimização do consumo de energia em residências pelo maior impacto visível ao meio ambiente (Congradac et al., 2012). Entretanto, a linha de pesquisa que estuda a dinâmica dos padrões de consumo de energia no sector residencial no mundo se consolidou nos últimos anos, tornando-se bastante popular o estudo e análise dessa temática com base em estudos de campo, estudos de caso, abordagens qualitativa e quantitativa, uso de inquéritos por questionários, olhando para vários tipos de combustíveis e, tomando como variáveis as características endógenas e exógenas dos agregados familiares, como bem ilustram os trabalhos de Eberhard (1990), Hansen (2000), Achão (2003), Tso e Yau (2003), Yohanis (2012), Luz (2014), Ofetotse et al. (2015), França (2017), Ateba et al. (2018), entre outros.

No estudo realizado por Eberhard (1990), sobre padrões de consumo de energia e problemas de abastecimento em áreas subdesenvolvidas na África do Sul, por exemplo, o autor focalizou em usos finais de energia nas zonas urbanas, peri-urbanas e rural, tendo mostrado que a energia nas zonas urbanas é usada para várias actividades. 0 estudo mostrou que devido a falta de fornecimento da energia eléctrica nas zonas peri-urbanas, maior parte dos agregados são forçados a gastarem valores consideráveis do seu orçamento para compra de combustíveis fósseis (petróleo, gás e carvão mineral) para atender parte das suas necessidades básicas, como é o caso de confecção de alimentos. Enquanto isso, em áreas rurais o estudo mostra que a demanda de lenha tem sido superior a oferta com consequências sociais, económicas e ambientais devastadoras.

Hansen (2000), realizou no Brasil um estudo similar analisando os padrões de consumo de energia em dez diferentes tipos de residências na Cidade de Porto Alegre, situada no Estado do Rio Grande do Sul, olhando apenas para o consumo de electricidade, tendo concluído que é possível estimar consumos futuros em áreas urbanas a serem ocupadas por residências semelhantes às estudadas. Ainda no Brasil Achão (2003) realizou um estudo sobre análise da estrutura de consumo de energia pelo sector residencial,

onde se verificou a redução do uso da lenha associada à penetração da electricidade e do GLP, além do aumento da participação de usos mais eficientes da energia eléctrica, como é o caso dos electrodomésticos em geral, aparelhos electrónicos e lâmpadas. Quanto às fontes, a electricidade e os derivados de petróleo passaram a desempenhar um papel de destaque no consumo energético brasileiro (Achão, 2003, p. 93-94).

Em Hong Kong, Tso e Yau (2003) investigaram como tipos de habitação, características da família (sexo, idade, nível de instrução, profissão e renda familiar, o número de membros do agregado familiar) e posse de aparelhos influência no consumo de energia nos lares. Os resultados do estudo indicaram uma forte ligação entre os parâmetros investigados e com efeitos significativos sobre o consumo de energia doméstica. Além disso, Yohanis (2012) observa no seu estudo como as posições sociais em 
relação à ocupação/status de emprego influência o consumo próprio de cada grupo, para ele, o emprego marca o nível de vida pelo seu nível de despesa.

Em Portugal, Luz (2014) realizou, também, um estudo similar no qual analisa os padrões de consumo de famílias portuguesas mediante abordagem qualitativa e um inquérito por questionário, olhando para electricidade, gás e parafina, tomando como variáveis o número de agregados familiares, nível de rendimento, idade, escolaridade. Os resultados desse estudo sugerem que os consumidores residenciais apresentam uma diversidade de hábitos no uso da energia e um dos maiores desafios é o de prever a demanda a fim de equacionar a oferta com o consumo.

Em um estudo feito em Botswana por Ofetotse et al. (2015), com base em combinação de dados medidos com levantamentos quantitativos, aponta que o número de pessoas influência na quantidade de energia em um uso doméstico e esta, não pode ser tratado como um factor independente ao avaliar o consumo de energia. Os resultados apresentados neste estudo indicam que o consumo de energia aumenta com o número de pessoas em uma casa, embora isso nem sempre é o caso. 0 estudo também indica que as famílias de Botswana apresentam padrões de uso de energia diferentes, que são únicas para cada agregado familiar. Para estes autores entender os padrões de consumo energético ajuda a influenciar a conservação reduzindo a escassez de energia aguda que Botswana está enfrentando actualmente.

França (2017), usando o método de estudo de caso e uma pesquisa do tipo exploratória, analisou o consumo energético de uma casa de alto padrão em Porto Velho, Estado Brasileiro de Rondónia, olhando para o uso de energia solar e eléctrica. Esta autora constatou que a energia é responsável por grande parte dos gastos mensais em casas de alto padrão. Esta constatação reforça a ideia de que sendo este um dos recursos mais utilizados pelo homem, o consumo consciente deste insumo é de extrema relevância do ponto de vista social, económico, tecnológico e ambiental, seja no âmbito global, nacional ou individual.

Um outro estudo realizado na África do Sul, por Ateba et al. (2018) analisa, nas famílias seleccionadas, os determinantes de escolha de combustível de energia para o consumo sustentável. Nesse estudo, constatou-se que a electricidade é a única forma de energia que as famílias utilizam universalmente para diversas actividades, como cozinhar, iluminação e aquecimento. A utilização de GPL é principalmente limitada a cozinhar, a energia solar para aquecimento de água. Por último, a biomassa (lenha e carvão vegetal) usa-se igualmente para aquecer, cozinhar, iluminar, entre outras funções.

Portanto, a partir dos estudos analisados, pode-se concluir que existem vários determinantes dos padrões de consumo doméstico de energia, dos quais destacam-se, tipos de combustíveis/fontes usadas, características dos agregados familiares, hábitos do consumidor, tipo de residência, posse de electrodomésticos entre outros aspectos. Essa constatação por si só, justifica a realização de estudos que permitam aferir os padrões de consumo doméstico de energia nas cidades moçambicanas com a finalidade de perceber as dinâmicas envolvidas para garantir suprimentos de energia adequados e confiáveis a médio e longo prazo.

\section{Considerações finais}

De acordo com o recorte metodológico adoptado o estudo realizado mostra um aumento crescente de artigos publicados sobre padrões de consumo de energia no sector residencial nas últimas três décadas.

A maior parte dos artigos publicados são de origem estrangeira, com particular enfoque para Brasil, com poucos artigos publicados internamente. Esses achados evidenciam que o uso de energia é um serviço essencial para o desenvolvimento das 
famílias as quais utilizam para diversas actividades, como cozinhar, iluminação, aquecer, entre outras funções.

Portanto, do levantamento feito conclui-se que os factores determinantes na formação dos padrões de consumo das famílias são dinâmicos, diversos e multidimensionais, dai a necessidade de estudos de campo/caso para o entendimento das dinâmicas envolvidas na construção desses padrões. Parte dos resultados obtidos neste artigo irão subsidiar os estudos sobre padrões de consumo doméstico referentes ao $14^{\circ}$ Bairro Nhaconjo, na Cidade da Beira no centro de Moçambique.

\section{Conflito de interesses}

Os autores declaram não haver conflito de interesses.

\section{Referências}

Abrão, M. A. P. A comunicação, a recepção e o consumo enquanto práticas culturais: um novo olhar. In: Baccega, M. A. Comunicação e culturas do consumo. São Paulo: Atlas, 2008.

Achão, C. C. L. Análise da estrutura de consumo de energia pelo sector residencial brasileiro. Rio de Janeiro: Universidade Federal do Rio de Janeiro, 2003. (Tese de doutorado).

Arthur, F.; Soliano, O.; Mariezcurrena, V. Estudo de avaliação de energias renováveis em Moçambique. Maputo: Banco Central de Moçambique, 2011. (Relatório anual).

Arthur, M.; Bond, C. A.; Wilson, B. Estimation of elasticities for domestic energy demand in $\begin{array}{lllll}\text { Mozambique. Energy } & \text { Economics, } & \text { v. 34, n. 2, } & \text { p. 398-409, }\end{array}$ https://doi.org/10.1016/j.eneco.2011.08.006

Ateba, B. B.; Johannes, J.; Fourie, E. The impact of energy fuel choice determinants on sustainable energy consumption of selected South African households. Journal of Energy in South African, v. 29, n. 3, p. 51-65. 2018. https://doi.org/10.17159/24133051/2018/v29i3a4714

Barquete, A. C. C.; Silva, N. P. 0 avanço e as melhorias com o uso de energias. São Carlos: Universidade de São Paulo, 2013. (Trabalho de conclusão de curso).

Bekhet, H. A.; Ivy-Yap, L. L. Highlighting energy policies and strategies for the residential sector in Malaysia. International Journal of Energy Economics and Policy, v. 4, n. 3, p. 448-456, 2014.

Bethonico, M. B. M. Impactos sócio ambientais da produção de carvão vegetal no norte de Minas Gerais: o caso de Montezuma. Anais do II Seminário Internacional sobre Desenvolvimento Regional. Santa Cruz do Sul, 2006.

Blackwell, R. D.; Miniard, P. W.; Engel, J. F. Comportamento do consumidor. São Paulo: Cencage Learning, 2008.

Burgsdorff, S. K. Moçambique energias renováveis em relatório nacional do ponto de situação. 2. ed. Maputo: ALER - Associação Lusófona de Energias Renováveis, 2017.

Canettieri, T.; Fernandez P. R.; Finelli R.; Pereira T. A atividade de carvoejamento e o risco a saúde da população de Rancho Novo, Caeté-MG. PEGADA - A Revista da Geografia do Trabalho, v. 14, n. 1, p. 257-281, 2013. https://doi.org/10.33026/peg.v14i1.1027 
Carvalho, A. A.; Alves, J. E. D. Padrões de consumo dos arranjos familiares e das pessoas que moram sozinhas no Brasil e em Minas Gerais: uma análise de gênero e renda. Anais do XIV Seminário sobre a Economia Mineira, Diamantina, 2010.

Castro, G. Mídia, consumo, globalização e contemporaneidade. In: Baccega, M. A. Comunicação e culturas do consumo. São Paulo: Atlas, 2008.

Cebula, R. J. US residential electricity consumption: The effect of states' pursuit of energy efficiency policie. Applied Economics Letters, v. 19, n. 15, p. 1499-1503, 2012. https://doi.org/10.1080/13504851.2011.637884

Cohen, C. A. M. J. Padrões de consumo: desenvolvimento, meio-ambiente e energia no Brasil. Rio de Janeiro: Universidade Federal do Rio de Janeiro, 2002. (Tese de doutorado).

Comini, R.; Clement, F.; Puente, F.; Orlandi, A.; Oliveira, I.; Lima, P.; Beirão, D. Eficiência energética nos edifícios residenciais. Lisboa: Intelligente Energy, 2008.

Congradac, V.; Prebiracevic B.; Jorgovanovic N.; Stanisic D. Assessing the energy consumption for heating and cooling in hospitals. Energy and Buildings, v. 48, p. 146154, 2012. https://doi.org/10.1016/j.enbuild.2012.01.022

Cortez, A. T. C. Da produção ao consumo: impactos sócio ambientais no espaço urbano. In: Ortigosa, S. A.; Guarnieri; Cortez, A. T. C. Consumo e desperdício as duas faces das desigualdades. São Paulo: Editora UNESP, 2009.

Dergiades, T.; Tsoulfidis, L. Revisiting residential demand for electricity in Greece: New evidence from the ARDL approach to cointegration analysis. Empirical Economics, v. 41, p. 511-531, 2011. https://doi.org/10.1007/s00181-010-0381-8

Ding, Q.; Cai, W.; Wang, P.; Sanwal, M. The relationships between household consumption activities and energy consumption in China: An input-output analysis from the lifestyle. Applied Energy, v. 207, p.520-532, 2017. https://doi.org/10.1016/j.apenergy. 2017.06.003

Dominik, E. C. Padrão de consumo familiar em diferentes estágios do ciclo de vida e níveis de renda-Bambuí-MG. Viçosa: Universidade Federal de Viçosa, 2010. (Dissertação de mestrado).

Dominik, E. C.; Silva, N. M.; Loreto, M. D. S.; Noronha, J. F. Padrão de consumo familiar em diferentes estágios de ciclo de vida e níveis de renda no Município de Bambuí-MG. Oikos: Família e Sociedade em Debate, v. 23, n. 1, p. 201-225, 2012.

Druckman, A.; Jackson, T. The carbon footprint of UK households 1990-2004: A socioeconomically disaggregated, quasi-multiregional input-output model. Ecological Economics, v. 68, p. 2066-2077, 2009. https://doi.org/10.1016/j.ecolecon.2009.01.013

Eberhard, A. A. Energy consumption patterns and supply problems in underdeveloped areas in South Africa. Development Southern Africa, v. 7, n. 3, p. 335-346, 1990. https://doi.org/10.1080/03768359008439525

ELETROBRAS - Centrais Elétricas Brasileiras S. A. Pesquisa de posse de equipamentos e hábitos de uso, ano base 2005: classe Residencial Relatório Brasil - Sumário Executivo. Rio de Janeiro: ELETROBRAS, PROCEL, 2009. (Avaliação do Mercado de Eficiencia Energética no Brasil). Disponível em: <http://www.procelinfo.com.br/main.asp?View= $\{$ 5A08CAF0-06D1-4FFE-B335-95D83F8DFB98 $\}$ Team $=$ Tms $=$ itemID $=\{99 E B B A 5 C-2 E A 1-$ 4AEC-8AF2-5A751586DAF9 $\} ; \& U I P a r t U I D=\{05734935-6950-4 E 3 F-A 182-$ 629352E9EB18\}>. Acesso em: 21 abr. 2020. 
Ferreira, A. M. S. Reconhecimento de padrões e tipificação de perfis de consumo: contribuições para a melhoria da gestão na distribuição da energia eléctrica. Salvador: Universidade Federal da Bahia, 2015. (Tese de doutorado).

Ferreira, J. Z. Estudo comparativo entre lâmpadas fluorescentes tubulares T8 e tubulares de LED. Curitiba: Universidade Tecnológica do Paraná, 2014. (Monografia de especialização).

França, V. S. Estudo de caso: análise do consumo energético de uma casa de alto padrão em Porto Velho, Rondônia. Palhoça: Universidade do Sul de Santa Catarina, 2017. (Trabalho de conclusão de curso).

Goldemberg, J.; Lucon, O. Energia e meio ambiente no Brasil. Estudos Avançados, v. 21, n. 59, p. 7-20, 2007. https://doi.org/10.1590/S0103-40142007000100003

Hansen, A. M. D. Padrões de consumo de energia eléctrica em diferentes tipologias de edificações residenciais, em Porto Alegre. Porto Alegre: Universidade Federal do Rio Grande do Sul, 2000. (Dissertação de mestrado).

IAEA - International Atomic Energy Agency. Energy indicators for sustainable development: Guidelines and methodologies. Viena: IAEA, 2005. Disponível em: <https://www.iaea.org/publications/7201/energy-indicators-for-sustainable-

development-guidelines-and-methodologies>. Acesso em: 21 abr. 2020.

Kemmler, A.; Spreng, D. Energy indicators for tracking sustainability in developing $\begin{array}{lllll}\text { countries. } & \text { Energy Policy, } & \text { v. 35, } & \text { n. } 4, & \text { p. 2466-2480, }\end{array}$ https://doi.org/10.1016/j.enpol.2006.09.006

Kowsari, R.; Zerriffi, H. Three-dimensional energy profile: A conceptual framework for assessing household energy use. Energy Policy, v. 39, n. 12, p.505-517, 2011. https://doi.org/10.1016/j.enpol.2011.06.030

La Rovere, E. L. Políticas de energia das famílias e mitigação climática: investigando práticas de energia no sector da habitação. Rio de Janeiro: Universidade Federal do Rio de Janeiro, 2015. v. 3 (Primeiro relatório de avaliação nacional).

Luz, N. M. G. F. Análise dos padrões de consumo de famílias portuguesas. Lisboa: Universidade Nova, 2014. (Dissertação de mestrado).

Mahumane, G.; Mulder, P. Mozambique energy Outlook, 2015-2030. Data, scenarios and policy implications research. Amsterdam: Faculty of Economy and Business Administration, 2015. (Research Memorandum 2015-7). https://doi.org/10.2139/ssrn.2644144

Manuel, M. T.; Victorino, A. Relações entre padrões de consumo doméstico de energia e níveis de renda familiar: estudo de caso no $14^{\circ}$ B Bairro Nhaconjo, Cidade da Beira, Moçambique. Revista Brasileira de Gestão Ambiental e Sustentabilidade, v. 7, n. 16, p. 619-631, 2020a. https://doi.org/10.21438/rbgas(2020)071611

Manuel, M. T.; Victorino, A. Padrões de consumo de energia no sector residencial: estudo de caso no 14을 Bairro Nhaconjo, Cidade da Beira, Moçambique. Revista Brasileira de Gestão Ambiental e Sustentabilidade, v. 7, n. 16, p.633-651, 2020b. https://doi.org/10.21438/rbgas(2020)071612

Mendes, N. J. A política energética no contexto das dinâmicas globais. Relações Internacionais (R:I), v. 28, p. 133-138, 2010. 
Ministério da Energia. Estratégia de desenvolvimento de energias novas e renováveis (EDENR) para o período de 2011-2025. Maputo: Ministério da Energia, 2011.

Moura, M. L. S.; Ferreira, M. C.; Paine, P. A. Manual de elaboração de projectos de pesquisa. Rio de Janeiro: EdUERJ, 1998.

Nkundumukiza, M. N. Queima de biomassa e os problemas de saúde dos habitantes do Distrito de Braço do Rio, Município de Conceição da Barra. Vitória: Universidade Federal do Espírito Santo, 2009. (Monografia de graduação).

Ofetotse, E. L.; Essah, E. A.; Yao, R. Trends in domestic electricity consumption in Botswana. TMC Academic Journal, v. 9, n. 2, p. 83-104, 2015.

Oliveira, M. F.; Viana A. R.; Santos M. O consumo alimentar de escolares: um artigo de revisão. Anais do IX Encontro Nacional de Pesquisa em Educação em Ciências - IX ENPEC, Águas de Lindóia, 2013.

Rocha, E.; Barros, C. Entre mundos distintos: notas sobre comunicação e consumo em um grupo social. In: Baccega, M. A. Comunicação e culturas do consumo. São Paulo: Atlas, 2008. p. 186-202.

Roger, A. H.; Merlin, K. Energia e meio ambiente. São Paulo: Cengage, 2010.

Scari, M. E. Cenários de consumo de energia elétrica para o setor residencial de Belo Horizonte. Belo Horizonte: Universidade Federal de Minas Gerais, 2011. (Dissertação de mestrado).

Sebastião, P. A. Modelo da electrificação de Moçambique: a importância do combate à desflorestação no meio rural. Lisboa: Instituto Supeiror de Gestão de Energias, 2013. (Dissertação de mestrado).

Silva, B. M. F.; Ferreira, O. M. K.; Anésio C. G. L.; Lopes, N. O.; Oliveira, T. O.; Dominik, C. E. Padrão de consumo das famílias urbanas de Bambuí-MG. Investidura: Portal Jurídico, 2017. Disponível em: <http://investidura.com.br/biblioteca-juridica/artigos/economia/ 336792-padrao-de-consumo-das-familias-urbanas-de-bambui-mg>. Acesso em: 21 abr. 2020.

Silva, A. L. G. R. Electricidade, crescimento económico e desenvolvimento: uma contribuição para o estudo do caso de Moçambique. Porto: Universidade do Porto, 2016. (Dissertação de mestrado).

Sousa, J. R. B.; Silva, S. M.; Almeida, M. G. Enquadramento energético do sector residencial português. Anais do Seminário Reabilitação Energética de Edifícios, Universdade do Minho, p. 49-56, 2012. Disponível em: $<$ http://repositorium.sdum.uminho.pt/bitstream/1822/21508/2/SREE-JS-SMSMA2.pdf>. Acesso em: 21 abr. 2020.

Tódero, M. Consumo consciente e percepção do consumidor sobre acções corporativas vinculadas ao conceito de responsabilidade social: um estudo no sector da saúde. Caxias do Sul: Universidade de Caxias do Sul, 2009. (Dissertação de mestrado).

Tso, G. K. F.; Yau, K. K. W. A study of domestic energy usage patterns in Hong Kong. Energy, v. 28, p. 1671-1682, 2003. https://doi.org/10.1016/S0360-5442(03)00153-1

Vasconcellos, M. A. S. Economia micro e macro. 3. ed. São Paulo: Atlas, 2002. 
Veiga Neto, A. R.; Souza, S. L. B.; Almeida, S. T.; Castro, F. N.; Braga Júnior, S. S. Factores que influenciam os consumidores da Geração Z na compra de produtos electrónicos. RACE Revista de Administração, Contabilidade e Economia, v. 14, n. 1, p. 287-312, 2014. https://doi.org/10.18593/race.v14i1.4935

Werlang, B. C. A.; Geller, I. Uma análise da relação entre o consumo de energia eléctrica e o crescimento econômico no mundo. Rio de Janeiro: Universidade Federal do Rio de Janeiro, 2018. (Projeto de graduação).

Yohanis, Y. G. Domestic energy use and householder's energy behaviour. Energy Policy, v. 41, p. 654-665, 2012. https://doi.org/10.1016/j.enpol.2011.11.028 